\title{
Implications of elemental abundances in dwarf spheroidal galaxies
}

\author{
T. Tsujimoto
}

National Astronomical Observatory, Mitaka-shi, Tokyo 181-8588, Japan

e-mail: taku.tsujimoto@nao.ac.jp

Received 30 August 2005 / Accepted 17 September 2005

\section{ABSTRACT}

Unusual elemental abundance patterns observed for stars belonging to nearby dwarf spheroidal (dSph) galaxies are discussed. Analysis of the $[\alpha / \mathrm{H}]$ vs. $[\mathrm{Fe} / \mathrm{H}]$ diagrams where $\alpha$ represents $\mathrm{Mg}$ or an average of $\alpha$-elements reveals that Fe from type Ia supernovae (SNe Ia) does not contribute to the stellar abundances in the dSph galaxies where the member stars exhibit low $\alpha / \mathrm{Fe}$ ratios except for the most massive dSph galaxy, Sagitarrius. The more massive dwarf (irregular) galaxy, the Large Magellanic Cloud, also has an SNe Ia signature in the stellar abundances. These findings suggest that whether SNe Ia contribute to chemical evolution in dwarf galaxies is likely to depend on the mass scale of galaxies. Unusual $\mathrm{Mg}$ abundances in some dSph stars are also found to be the origin of the large scatter in the $[\mathrm{Mg} / \mathrm{Fe}]$ ratios and are responsible for a seemingly decreasing $[\mathrm{Mg} / \mathrm{Fe}]$ feature with increasing $[\mathrm{Fe} / \mathrm{H}]$. In addition, the lack of massive stars in the dSph galaxies does not satisfactorily account for the low- $\alpha$ signature. Considering the assembly of deficient elements $(\mathrm{O}, \mathrm{Mg}, \mathrm{Si}, \mathrm{Ca}, \mathrm{Ti}$ and $\mathrm{Zn})$, all of which are synthesized in pre-SN massive stars and in SN explosions, the low- $\alpha$ signature appears to reflect the heavy-element yields of massive stars with less rotation compared to solar neighborhood stars.

Key words. galaxies: abundances - galaxies: evolution - nuclear reactions, nucleosynthesis, abundances - stars: abundances stars: rotation - stars: supernovae: general

\section{Introduction}

The elemental abundance patterns of stars reflect the combination of nucleosynthesis yields and star formation histories. Now that it is possible to obtain detailed elemental abundances of individual stars beyond the Milky Way thanks to ongoing observations by $8-\mathrm{m}$ class telescopes, nearby galaxies can be used as a laboratory to check the information accumulated to date through the numerous studies on stars in the Milky way (e.g., Wheeler et al. 1989; McWilliam 1997). Such validation will also address the issue of whether the knowledge obtained for our galaxy can be applied to the chemical evolution of other galaxies, and represents a step toward investigation of the distant universe, where information on elemental abundances remains limited and uncertain.

At present, the theoretical understanding of the observed elemental abundance patterns of stars in nearby dwarf spheroidal (dSph) galaxies has come to a standstill. One of the most remarkable abundance features in these galaxies revealed by accumulated observational results (Shetrone et al. 2001, 2003; Bonifacio et al. 2004; Geisler et al. 2005; Monaco et al. 2005) is the deficiency of $\alpha$-elements in comparison with Fe. At a first glance, knowledge derived for the solar neighborhood provides a reasonable explanation for the origin of the observed low- $\alpha$ signature as being due to the additional supply of $\mathrm{Fe}$ from type Ia Supernovae (SNe Ia). Based on this supposition, theoretical models of evolutionary change in $[\alpha / \mathrm{Fe}]$ against
$[\mathrm{Fe} / \mathrm{H}]$ in $\mathrm{dSph}$ galaxies have been proposed (Ikuta \& Arimoto 2002; Lanfranchi \& Matteucci 2004; Robertson et al. 2005). However, there is no compelling evidence for the contribution of SNe Ia to other elemental ratios such as $[\mathrm{Mn} / \mathrm{Fe}]$ or [n-capture/Fe] (see Tsujimoto \& Shigeyama 2002). The dSph stars thus exhibit unusual elemental abundance patterns that cannot be explained by current knowledge (see also Shigeyama \& Tsujimoto 2003).

The chemical evolution of stars is often discussed in terms of the relative abundance ratios between heavy elements, against conventionally $[\mathrm{Fe} / \mathrm{H}]$. The relative abundance of an element $\mathrm{X}$ with respect to iron $([\mathrm{X} / \mathrm{Fe}])$ is a typical example. In most cases, the $[\mathrm{X} / \mathrm{Fe}]$ values are confined to much less than 1 dex. Due to the small range of the distributed values, intrinsic scatter partly due to observational error often obscures the fundamental abundance trend. The alternative diagram of two ratios with respect to hydrogen, such as $[\mathrm{X} / \mathrm{H}]$ vs. $[\mathrm{Fe} / \mathrm{H}]$, allows the overall abundance features in the range over $\sim 2$ dex to be resolved more clearly.

The evolution of $[\mathrm{Fe} / \mathrm{H}]$ is examined in this study as a function of $[\alpha / \mathrm{H}]$ in the dSph galaxies, and the results are compared with the data for the Milky Way and the Large Magellanic Cloud (LMC). The LMC is another nearby galaxy that hosts a number of red giants, which also exhibit lower $[\mathrm{O} / \mathrm{Fe}]$ ratios than stars in the solar neighborhood at the same $[\mathrm{Fe} / \mathrm{H}]$ (Smith et al. 2002). This analysis reveals a clear contribution 


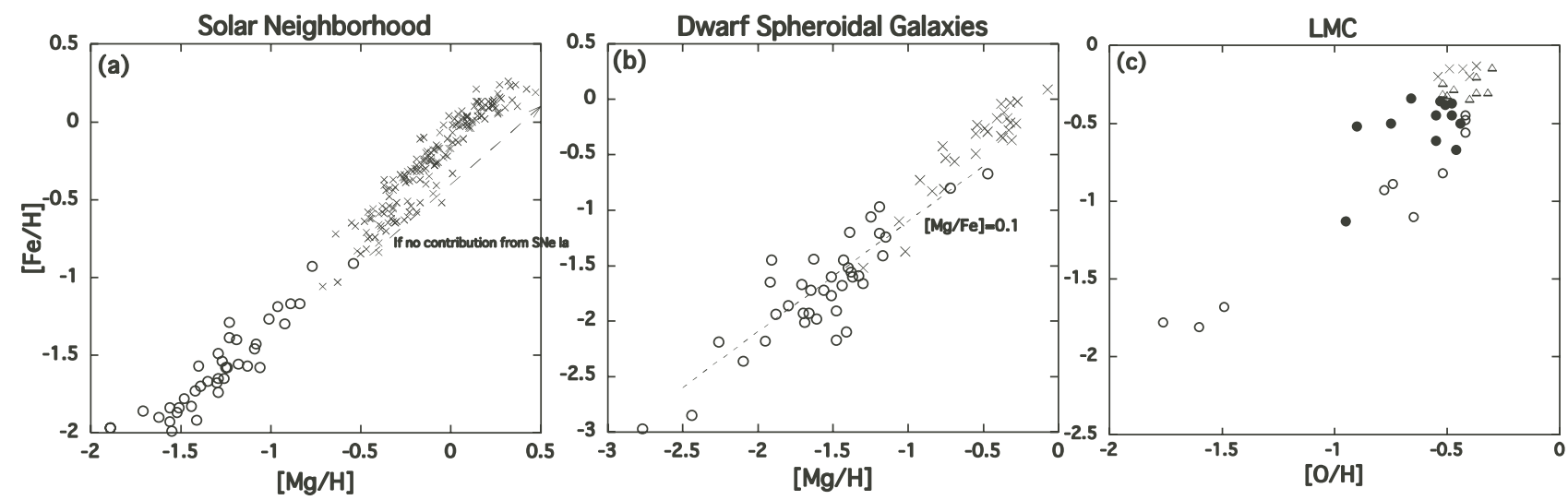

Fig. 1. a) Fe and Mg abundances for solar neighborhood stars (open circles, Hanson et al. 1998; crosses, Edvardsson et al. 1993). Dashed arrow denotes the evolutionary path expected without contribution from SNe Ia. b) Fe and Mg abundances for dSph stars, showing Draco, Ursa Minor, Sextan, Sculptor, Carina, Fornax, and Leo I (open circles) (Shetrone et al. 2001, 2003; Geisler et al. 2005), and Sagittarius (crosses) (Bonifacio et al. 2004; Monaco et al. 2005). Dotted line denotes the correlation $[\mathrm{Mg} / \mathrm{Fe}]=0.1$. c) $\mathrm{Fe}$ and $\mathrm{O}$ abundances for LMC stars, showing observed data from Smith et al. (2002) (filled circles), Hill et al. (2000) (open circles), Hill et al. (1995) (triangles), and Korn et al. (2002) (crosses).

from SNe Ia in the LMC stars as well as stars belonging to the Sagittarius (Sgr) galaxy, but not for stars in other smaller mass dSph galaxies. This is reinforced by analysis of the Mn abundances.

The origin of the low $\alpha / \mathrm{Fe}$ ratios for the $\mathrm{dSph}$ stars is then discussed. The lack of massive stars of greater than $\sim 20 M_{\odot}$ in the $\mathrm{dSph}$ galaxies is proposed as a potential reason for the low $\alpha / \mathrm{Fe}$ ratios, as the combination of theoretical nucleosynthesis yields for $\alpha$-elements and the Fe mass inferred from SN-light curve analyses predicts that an ensemble of exclusively lowmass type-II SNe (SNe II) results in low $[\alpha / \mathrm{Fe}]$. However, it is shown through abundance studies based on $n$-capture elements and $\mathrm{Zn}$ that this possibility is unlikely.

The discussion thus reduces to the features of nucleosynthesis in SNe II. The dSph stars exhibit deficiencies in the abundances of not only $\alpha$-elements (O to Ti) but also $\mathrm{Zn}$. Theoretical nucleosynthesis calculations have revealed that some of the depletions arise in pre-SN massive stars, while other deficiencies reflect modification or synthesis in the final SN II explosions (Pagel 1997, and references therein). This suggests that a mechanism modifying all stages in the life of massive stars and the corresponding SN yields might be required. This scenario is reminiscent of stellar rotation, which may change both the onion-skin structure during stellar evolution (Heger et al. 2000; Heger \& Langer 2000; Hirschi et al. 2004) and the form of the eventual SN explosions (e.g., Fryer \& Warren 2004). It can thus be speculated that the rotation is affected by the environment of stars at birth in such a way that dSph stars develop lesser rotation than in the solar neighborhood stars.

\section{2. $[\mathrm{Fe} / \mathrm{H}]$ vs. $[\alpha / \mathrm{H}]$ diagrams}

This analysis starts with an investigation of whether the low $\alpha / \mathrm{Fe}$ ratios in the $\mathrm{dSph}$ stars are promoted by contributions from SNe Ia. If the Fe supply from SNe Ia is added to interstellar matter that has been already enriched by SNe II, the slope of $[\mathrm{Fe} / \mathrm{H}]$ against $[\mathrm{Mg} / \mathrm{H}]$ will steepen from the onset of SNe Ia. Thus, after SNe Ia start to contribute, the increase in $[\mathrm{Fe} / \mathrm{H}]$ becomes larger than that in $[\mathrm{Mg} / \mathrm{H}]$, while $[\mathrm{Mg} / \mathrm{Fe}]=$ const. until the occurrence of SNe Ia. A good reference for the resolution of such a tendency is the relation for the solar neighborhood, where SNe Ia have been confirmed to contribute to chemical evolution (e.g., Matteucci \& Greggio 1986). Figure 1a shows the $[\mathrm{Fe} / \mathrm{H}]$ vs. $[\mathrm{Mg} / \mathrm{H}]$ diagram for the solar neighborhood stars. As expected, the slope changes at $[\mathrm{Fe} / \mathrm{H}] \sim-1$ and the bending feature is seen for disk stars, confirming the additional supply of Fe from $\mathrm{SNe}$ Ia, although a small fraction of stars belong to the thick disk and are distributed on the extension of a trend of halo stars at $[\mathrm{Fe} / \mathrm{H}] \gtrsim-1$ (Feltzing et al. 2003).

In contrast to the Milky Way, there appears to be essentially no change in the slope of the relation between $[\mathrm{Fe} / \mathrm{H}]$ and $[\mathrm{Mg} / \mathrm{H}]$ for the seven $\mathrm{dSph}$ galaxies, i.e., Draco, Ursa Minor, Sextan, Sculptor, Carina, Fornax, and Leo I (open circles; Fig. 1b). The bending feature as evidence of SNe Ia appearance is not seen over the range of $-3 \lesssim[\mathrm{Fe} / \mathrm{H}] \lesssim-0.5$. The observed data are distributed along the line $[\mathrm{Mg} / \mathrm{Fe}]=0.1$, i.e., $\Delta[\mathrm{Mg} / \mathrm{H}] / \Delta[\mathrm{Fe} / \mathrm{H}]=1$, indicative of no Fe supply from SNe Ia. Although the scatter about the line $[\mathrm{Mg} / \mathrm{Fe}]=0.1$ is prominent and it renders the contribution of SNe Ia to chemical evolution difficult to judge from this analysis, the absence of such contributions is supported by the Mn abundances, as discussed in the next section. On the other hand, the stars belonging to the Sgr galaxy (crosses; Fig. 1b) appear to exhibit an elemental feature containing the signature of $\mathrm{Fe}$ input from $\mathrm{SNe}$ Ia, with some clear similarities to the solar neighborhood stars in Fig. 1a. It may imply that the massive Sgr galaxy compared to other dSph galaxies is likely to possess a gravitational potential well that is deep enough for $\mathrm{SNe}$ Ia to contribute to chemical evolution. However, a feature common to all the dSph stars is the low $\mathrm{Mg} / \mathrm{Fe}$ ratio without a clear contribution from SNe Ia.

The same conclusions for these $\mathrm{dSph}$ galaxies can be also deduced from the $[\mathrm{Fe} / \mathrm{H}]$ vs. $[\alpha / \mathrm{H}]$ diagrams where $\alpha=$ $\frac{1}{3}(\mathrm{Mg}+\mathrm{Ca}+\mathrm{Ti})$ (due to a lack of $\mathrm{Ti}$ data, $\mathrm{Si}$ is used for the $\alpha$ estimate in Bonifacio 2004), as shown in Fig. 2a. These $\alpha$ elements all have similar behaviors in the $[\alpha / \mathrm{Fe}]$ vs. $[\mathrm{Fe} / \mathrm{H}] \mathrm{di}-$ agrams for solar neighborhood stars, and therefore the average ratio of these elements to $\mathrm{Fe}$ is also a good indicator of the 

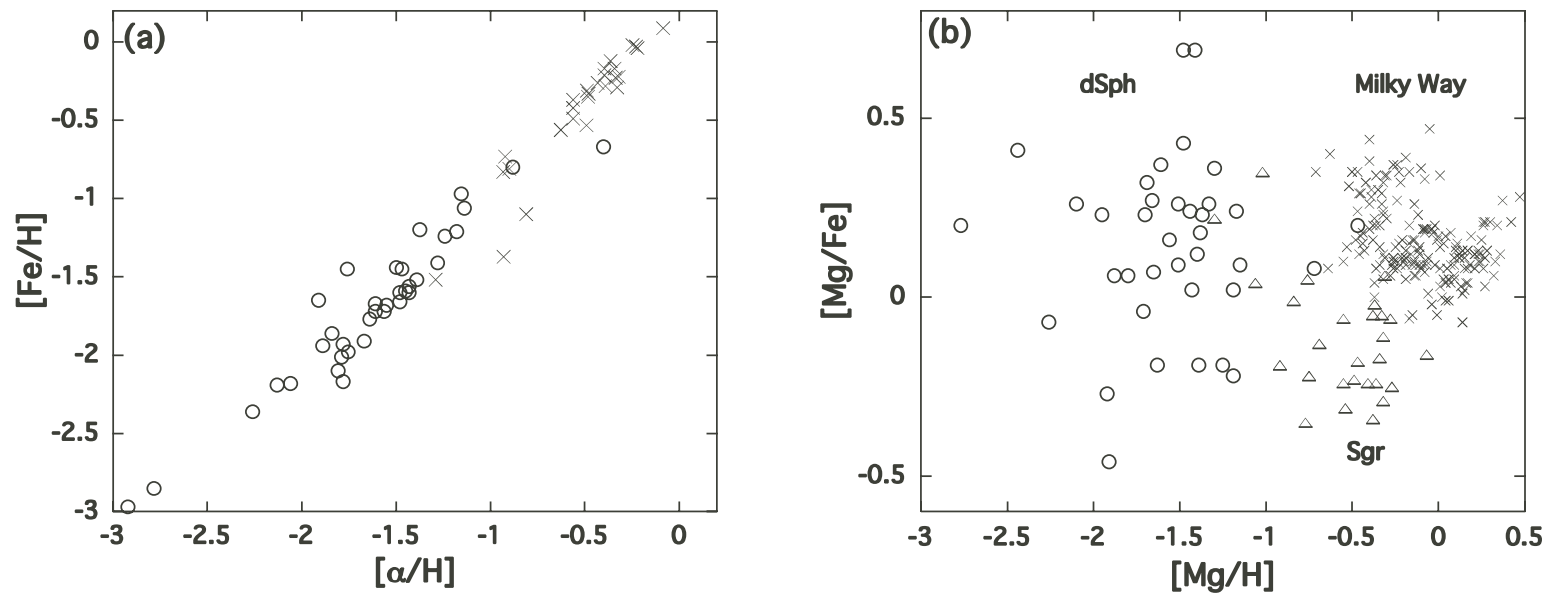

Fig. 2. a) Fe and $\alpha$ abundances for dSph stars, showing Draco, Ursa Minor, Sextan, Sculptor, Carina, Fornax, and Leo I (open circles) (Shetrone et al. 2001, 2003; Geisler et al. 2005), and Sagittarius (crosses) (Bonifacio et al. 2004; Monaco et al. 2005). Here $\alpha$ is an average of Mg, Ca, and $\mathrm{Ti}$, but $\mathrm{Si}$ is used for the $\alpha$ estimate in Bonifacio et al. (2004) due to a lack of Ti data. $\mathbf{b})[\mathrm{Mg} / \mathrm{Fe}]$ as a function of $[\mathrm{Mg} / \mathrm{H}]$ for dSph stars (open circles, Shetrone et al. 2001, 2003; Geisler et al. 2005), Sgr stars (open triangles, Bonifacio et al. 2004; Monaco et al. 2005), and disk stars in the Milky Way (crosses, Edvardsson et al. 1993).

relative contributions of SNe Ia and SNe II, although each $\alpha$ element has a different production process in SNe II, as will be discussed in Sect. 4.2. Figure $2 b$ offers another view on this subject. The seemingly decreasing $[\mathrm{Mg} / \mathrm{Fe}]$ features with the increasing metallicity $[\mathrm{Fe} / \mathrm{H}]$ for dSph stars as well as disk stars in the Milky Way (e.g., Shetrone et al. 2001, 2003; Bonifacio et al. 2004; Edvardsson et al. 1993) should be much the same as in the alternative case with the metallicity $[\mathrm{Mg} / \mathrm{H}]$ as long as these features are the end results of SNe Ia contribution. Despite a narrower metallicity range given by $[\mathrm{Mg} / \mathrm{H}] \mathrm{com}$ pared with $[\mathrm{Fe} / \mathrm{H}]$ which makes abundance trends of $[\mathrm{Mg} / \mathrm{Fe}]$ unclear, decreasing $[\mathrm{Mg} / \mathrm{Fe}]$ features are evidently seen for disk stars and Sgr stars. In contrast, this is not the case for stars in seven dSph galaxies, showing no sign of an $\mathrm{SNe}$ Ia contribution to the stellar abundances.

The absence of the contribution from SNe Ia in dSph galaxies except for the Sgr galaxy means that both $\alpha$-elements and $\mathrm{Fe}$ are synthesized only in SNe II and thereby their relative evolutions are not affected by variations in star formation history. On the other hand, in other cases that SNe Ia contribute to chemical evolution, the differential chemical enrichment between SNe II and SNe Ia caused by the difference in the abundance patterns of heavy elements ejected through SN explosions as well as the differential evolutionary timescale of the progenitors will make various stellar abundance patterns reflect the variations in star formation history of galaxies. One good example demonstrating it is the LMC as shown in Fig. 1c. The LMC stars exhibit an increase in $[\mathrm{Fe} / \mathrm{H}]$ against a considerably lower $\Delta[\mathrm{O} / \mathrm{H}]$ for $[\mathrm{Fe} / \mathrm{H}] \gtrsim-1([\mathrm{O} / \mathrm{H}]$ is used for the $\mathrm{LMC}$ due to a lack of observational data). In some stars, the $\mathrm{O}$ and $\mathrm{Fe}$ abundances appear to decrease, resulting in a bending feature pointing in the opposite direction to the cases of disk stars and Sgr stars. This somewhat complicated abundance behavior is interpreted as representing the end result of Fe supply from SNe Ia combined with a star formation history that differs from that in the solar neighborhood, suggesting the importance of galactic wind in a small galaxy (Venn et al. 2004) and/or a gap in star formation
(Gilmore \& Wyse 1991; Tsujimoto et al. 1995). Considering the masses of dwarf galaxies that exhibit an $\mathrm{SNe}$ Ia signature in stellar abundances, i.e., the LMC $\left(5.3 \times 10^{9} M_{\odot}\right.$; Alves \& Nelson 2000) and the Sgr galaxy $\left(2-5 \times 10^{8} M_{\odot}\right.$; Law et al. 2005), in comparison with other dSph galaxies with a mass in the range of several $10^{6}-10^{7} M_{\odot}$ (Mateo 1998), the condition of whether SNe Ia contribute to chemical evolution or not is likely to depend on the mass scale of galaxies.

It has been considered that the $\mathrm{dSph}$ galaxies have complex star formation histories, many of which have prolonged star formation over several Gyrs, from the viewpoint of their color-magnitude diagrams (Mateo 1998; Tolstoy et al. 2003; Venn et al. 2004; Dolphin et al. 2005). Such an inferred large span of ages (see also Grebel \& Gallagher 2004) may favor the possibility of the contribution of SNe Ia in the dSph galaxies. However, there is the possibility that the ejecta of SNe Ia easily escape from the galaxy due to inefficient cooling by a low density of gas, resulting in little/no contribution of SNe Ia to chemical evolution in spite of prolonged star formation. DSph galaxies may have formed through a prolonged accretion over Gyrs of small satellites, in each of which star formation continued for a period of several hundred million years. Thus, a large range of stellar ages does not necessarily imply an involvement of SNe Ia in the stellar abundances.

The spectroscopy of large numbers of stars in the Sculptor galaxy has been performed (Tolstoy et al. 2004) and an initial result on the $\alpha$-abundances (average of $\mathrm{Ca}, \mathrm{Mg}$, and $\mathrm{Ti}$ ) in comparison to Fe has been reported (Tolstoy 2005). This apparent decreasing $[\alpha / \mathrm{Fe}]$ feature with increasing $[\mathrm{Fe} / \mathrm{H}]$ may recall the $\mathrm{SNe}$ Ia contribution and therefore abundance data for each element including $\alpha$-elements will be required for further analyses. However, the $[\mathrm{Ba} / \mathrm{Fe}]$ ratios for these stars coincide with those of halos stars in the Milky Way at the same $[\mathrm{Fe} / \mathrm{H}]$ (Venn \& Hill 2005). This coincidence is at odds with an SNe Ia signature in the stellar abundances since the Fe supply from SNe Ia combined with the production of Ba synthesized through $s$-process that is expected to be similar at the 

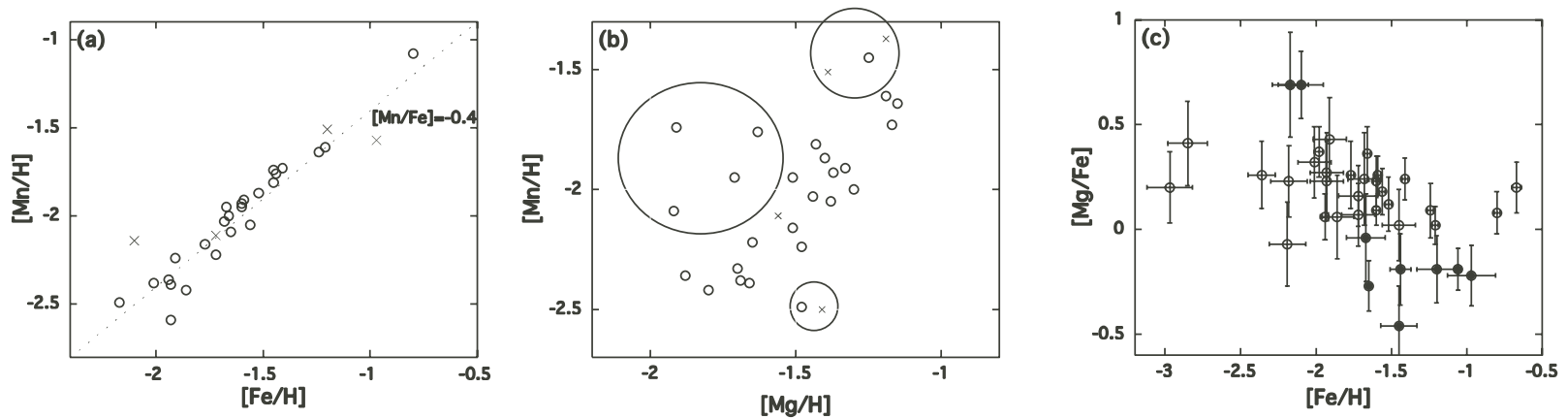

Fig. 3. a) Mn and Fe abundances for dSph stars (open circles, Shetrone et al. 2001, 2003; crosses, Geisler et al. 2005), including those for globular cluster Palomar 12 which is suggested to originate from the Sgr galaxy (Cohen 2004). Dotted line denotes the correlation [Mn/Fe] = -0.4. b) $\mathrm{Mn}$ and $\mathrm{Mg}$ abundances for $\mathrm{dSph}$ stars (unusual $\mathrm{Mg}$ abundances are outlined). The same symbols as for a). For three stars which largely deviate from the line $[\mathrm{Mn} / \mathrm{Fe}]=-0.4$ in a), their $\mathrm{Mn}$ abundances are corrected to have $[\mathrm{Mn} / \mathrm{Fe}]=-0.4$. c) $[\mathrm{Mg} / \mathrm{Fe}]$ as a function of $[\mathrm{Fe} / \mathrm{H}]$ with error bars taken from the literatures. Filled circles denote the stars highlighted in $\mathbf{b})$. Note that no Mn abundance data is available for some stars of the denoted by open circles.

same metallicity would lead to lower $[\mathrm{Ba} / \mathrm{Fe}]$ ratios than the observed data of halo stars. This result is compatible with previous reports (Shetrone et al. 2001, 2003), and has been one of the characteristic abundance features that dSph stars exhibit.

\section{Mn tracer in the dSph galaxies}

The Mn abundance is another important indicator to distinguish products of SNe Ia from those of SNe II; other Fe-group elements do not possess such a property. As shown in Fig. 3a, the Mn abundance of the dSph galaxies exhibits a strong correlation with the $\mathrm{Fe}$ abundance, forming a line of $[\mathrm{Mn} / \mathrm{Fe}] \sim-0.4$. For the Sgr galaxy, the data for the globular cluster Palomar 12 $([\mathrm{Fe} / \mathrm{H}]=-0.8)$, which is suggested to originate from this galaxy, is shown (Cohen 2004). The detailed $[\mathrm{Mn} / \mathrm{Fe}]$ feature for the Sgr galaxy is shown in McWilliam \& Smecker-Hane (2003), revealing that the $\mathrm{Mn} / \mathrm{Fe}$ ratios have an SNe Ia signature in the metal-rich regime, which is consistent with the analysis of $\alpha$-elements in Sect. 2. The Mn-Fe correlation in Fig. 3a implies that SNe Ia have not contributed to the Mn and $\mathrm{Fe}$ abundances since the $[\mathrm{Mn} / \mathrm{Fe}]$ ratio would become greater than -0.4 if $\mathrm{SNe}$ Ia contributed to $\mathrm{Mn}$ and $\mathrm{Fe}$ in these low$\alpha$ stars (Shetrone et al. 2003; Shigeyama \& Tsujimoto 2003). Furthermore, the strength of this correlation can be attributed to the monotonic increase in both $\mathrm{Fe}$ and $\mathrm{Mn}$ with time, representing good "nucleosynthesis clocks" in the dSph galaxies.

In contrast, the Mn abundance does not correlate with the $\mathrm{Mg}$ abundance (Fig. 3b). Since the Mg abundance should also increase monotonically with time similarly to $\mathrm{Fe}$ or $\mathrm{Mn}$, irrespective of variations in star formation history, anomalous $\mathrm{Mg}$ abundances for some stars are considered to obscure such increasing trends. The observed data that deviates substantially from the Mg-Mn correlation is indicated in the figure, and the same stars can be seen to exhibit unusual $[\mathrm{Mg} / \mathrm{Fe}]$ values with a seeming tendency to decrease with increasing $[\mathrm{Fe} / \mathrm{H}]$ (filled circles in Fig. 3c). The less tight correlation between Mn and $\mathrm{Mg}$ abundances as a result of excluding the data with unusual $\mathrm{Mg}$ abundances may result partially from the relatively large error $\sim 0.2$ dex for the $\mathrm{Mg}$ abundances, whereas a typical error of $\sim 0.1 \mathrm{dex}$ is seen for the $\mathrm{Fe}$ and $\mathrm{Mn}$ abundances. The anomaly in the $\mathrm{Mg}$ abundances for the stars far exceeds measurement errors. Therefore it is concluded that unusual $\mathrm{Mg}$ abundances in some $\mathrm{dSph}$ stars are the origin of the large scatter in the $[\mathrm{Mg} / \mathrm{Fe}]$ ratios and responsible for a seemingly decreasing $[\mathrm{Mg} / \mathrm{Fe}]$ with increasing $[\mathrm{Fe} / \mathrm{H}]$.

\section{The origin of low- $\alpha$ in dSph galaxies}

\subsection{Truncated initial mass function}

Theoretical nucleosynthesis models predict the synthesized mass of Mg in SN II progenitors to a certain extent, whereas the predicted mass of Fe depends on the mass cut chosen for the stellar core and is thus far less certain (e.g., Thielemann et al. 1990). Therefore, the $[\mathrm{Mg} / \mathrm{Fe}]$ ratio as a function of the progenitor mass $M_{\mathrm{ms}}$ cannot be determined purely from a theoretical viewpoint. Here, two approaches supplemented by observational data are employed to derive this relationship. The first approach is based on the abundance patterns of very metal-poor stars, for which recent studies have revealed that stars with elemental abundances of $-4.0 \lesssim[\mathrm{Fe} / \mathrm{H}] \lessgtr-2.5$ may have inherited the abundance pattern of the ejecta of the preceding $\mathrm{SNe}$ (Audouze \& Silk 1995; Shigeyama \& Tsujimoto 1998) or the products of the mixing of matter from bursts of the early $\mathrm{SNe}$ (Cayrel et al. 2004). Following these hypotheses, observational data (McWilliam et al. 1995; Cayrel et al. 2004) indicate no signs of SNe II with $[\mathrm{Mg} / \mathrm{Fe}] \sim 0-0.1$.

Alternatively, on the basis of Fe masses estimated from SN light-curve analyses, the Fe mass increases with increasing $M_{\mathrm{ms}}$ for $M_{\mathrm{ms}} \gtrsim 20 M_{\odot}$, yet remains nearly constant for $M_{\mathrm{ms}} \lesssim 20 M_{\odot}$ (Shigeyama \& Tsujimoto 1998; Maeda \& Nomoto 2003). As the synthesized Mg mass increases with increasing $M_{\mathrm{ms}}$, the breakdown of the linear relationship between the synthesized $\mathrm{Fe}$ mass and $M_{\mathrm{ms}}$ gives rise to low $\mathrm{Mg} / \mathrm{Fe}$ ratios for low-mass SNe II. For example, SNe II with $M_{\mathrm{ms}}=13,15 M_{\odot}$ give $[\mathrm{Mg} / \mathrm{Fe}]=-0.6,0$, respectively (Umeda \& Nomoto 2002). If this is the case, the lack of SNe II with $M \gtrsim 20 M_{\odot}$ is consistent with the low $\alpha / \mathrm{Fe}$ ratios for $\mathrm{dSph}$ 
stars (Venn et al. 2004). It should be noted, however, that the Fe mass estimate by this method has been applied to only a small number of SNe II. In addition, the estimation involves uncertainties due to a dependence on the distance to the galaxies hosting the $\mathrm{SNe}$ and uncertain prediction of the progenitor mass (e.g., Van Dyk et al. 2002). Such a truncated initial mass function (IMF) in the dSph galaxies is at odds with other features as follows.

1. Similarity of $n$-capture/Fe ratios. The two neutron capture processes, $s$ - and $r$-processes, operate in different astrophysical sites according to different physical conditions; the $s$-process occurs during mixing episodes in low-mass asymptotic giant branch (AGB) stars, whereas the $r$-process may occur during SN II explosions of progenitors within a narrow mass range such as $8-10 M_{\odot}$ (e.g., Travaglio et al. 1999; Ishimaru \& Wanajo 1999) or 20-25 $M_{\odot}$ (Tsujimoto et al. 2000). If SNe with 8-10 $M_{\odot}$ are assumed for the production site of $r$-process, the truncation of massive stars above $\sim 20 M_{\odot}$ will not affect any of the yields of the $n$-capture elements, while the yields of $\alpha$ - and Fe-group elements integrated over all SNe II will certainly decrease. Taking the Salpeter IMF and setting the truncation at $M=$ $15 M_{\odot}$, the Fe production rate per generation of stars is reduced by nearly half, resulting in a $\sim 0.3$ dex offset in [ $n$-capture/Fe] between dSph stars and solar neighborhood stars. However, the observed data do not support such differences, and rather suggest similar ratios (Shetrone et al. 2001, 2003; Venn et al. 2004; Venn \& Hill 2005). Similarly, the choice of 20-25 $M_{\odot}$ SNe for the $r$-process site is obviously incompatible with the observation since the production of $r$-process elements hardly occurred in the dSph galaxies with the truncated IMF. The large uncertainty in the production sites of the $s$ - and $r$-processes, including the potential production of $r$-process elements through merging neutron stars and the operation of the weak $s$-process in massive stars, make discussion of the above offset rather complicated. However, a difference in the [ $n$-capture/Fe] ratios between $\mathrm{dSph}$ stars and solar neighborhood stars is unavoidable, given the truncated IMF.

2. Low $\mathrm{Zn} / \mathrm{Fe}$ ratio. Zinc is also deficient in $\mathrm{dSph}$ galaxies (Shetrone et al. 2001, 2003; Sbordone 2005). The [Zn/Fe] ratios for solar neighborhood stars are basically above the solar ratio (e.g., Cayrel et al. 2004; Nissen et al. 2004), whereas dSph stars exhibit $[\mathrm{Zn} / \mathrm{Fe}] \sim-0.3$ on average with large scatter. Although there is substantial ambiguity regarding the site of $\mathrm{Zn}$ production (e.g., Chen et al. 2004), the high $[\mathrm{Zn} / \mathrm{Fe}]$ ratios of very metal-poor stars (Cayrel et al. 2004) strongly suggest that SNe II are prominent sites. It has been proposed that effective $\mathrm{Zn}$ production can be realized in SNe with large energies (i.e., hypernovae, Umeda $\&$ Nomoto 2002). Their calculations reveal that low-mass $\mathrm{SNe}$ with low $[\alpha / \mathrm{Fe}]$ ratios produce high $[\mathrm{Zn} / \mathrm{Fe}]$ ratios of $\sim 0.1-0.6$, depending on the explosion energy. This result is incompatible with the formation of low $[\mathrm{Zn} / \mathrm{Fe}]$ ratios due to the truncated IMF. The high $[\mathrm{Zn} / \mathrm{Fe}]$ ratios in low-mass $\mathrm{SNe}$ are due to the relatively high $\mathrm{Fe}$ production $\left(\sim 0.07 M_{\odot}\right)$ assumed in theoretical models, which requires a large Si-burning layer where $\mathrm{Zn}$ is produced. In other words, low $[\alpha / \mathrm{Fe}]$ ratios necessarily yield high $[\mathrm{Zn} / \mathrm{Fe}]$ ratios in current models.

\subsection{Stellar rotation}

The elements with clearly deficient abundances with respect to Fe in dSph stars, compared to halo stars in the solar neighborhood, are $\mathrm{O}, \mathrm{Na}, \mathrm{Mg}, \mathrm{Al}, \mathrm{Si}, \mathrm{Ca}, \mathrm{Ti}$ and $\mathrm{Zn}$. Here, $\mathrm{Na}$ and $\mathrm{Al}$ are secondary elements, the production of which is limited by the small amount of seed heavy elements ( $\mathrm{Ne}$ and $\mathrm{Mg}$ ) contained in the initial chemical composition of the SN progenitor star (see also Venn et al. 2004). The mechanism modifying the SN yields of the remaining elements ( $\alpha$-elements and $\mathrm{Zn}$ ) is postulated as follows. The elements $\mathrm{O}$ and $\mathrm{Mg}$ are produced as a result of hydrostatic burning in massive stars, and SN explosions merely expel these elements without affecting their yields. In contrast, the yields of $\mathrm{Si}$ and $\mathrm{Ca}$ are modified by the final $\mathrm{SN}$ explosions, with changes beginning from the formation of the onion-skin structure. Titanium and $\mathrm{Zn}$ are also produced in explosive nucleosynthesis. Therefore, the process causing certain elements to be produced less effectively in dSph stars compared to solar neighborhood stars must be active in both pre-explosive massive stars and during SN explosions.

Stellar rotation has the potential to produce the observed variety in the yields of all these elements. The effects of rotation on the evolution of pre-SN massive stars have been discussed in detail (Hirschi et al. 2004). Rotation-induced mixing, by which the products of the burning in the core are mixed into the stellar envelope and new fuel is supplied to the convectively burning stellar core, has been shown to have a marked impact on the internal structure, even altering the deep interior of the core (e.g., mass of the Si core). The calculations by Hirschi et al. (2005) suggest that the yields of $\mathrm{C}$ and $\mathrm{O}$ are enhanced by stellar rotation. The $\mathrm{Mg}$ yield in a model with $M_{\mathrm{ms}}=12 M_{\odot}$ also increases substantially. Although these results are for stars with solar metallicity, Maeder \& Meynet (2005) reported that for stars with low metallicity, the effects of rotational mixing are also significant, and thus the yields of heavy elements such as $\mathrm{C}$ and $\mathrm{O}$ are enhanced by stellar rotation. Additionally, rotation has been suggested to promote deformed or asymmetric SN explosions, which are likely to involve jet-like explosions (e.g., Yamada \& Sato 1994). Asymmetric explosions have been found by Nagataki et al. (1997) to enhance the production of elements with mass numbers around $A=45$ (i.e., $\mathrm{Ca}$ and Ti) and more significantly around $A=65$ (i.e., $\mathrm{Zn}$ ) (see also Maeda \& Nomoto 2003).

This suggests that stars in the primeval $\mathrm{dSph}$ galaxies may have lower rotation than that of stars belonging to the Milky Way, at least for massive stars. If this is the case, massive dSph stars would produce relatively small amounts of $\alpha$-elements during stellar evolution, and end in spherical SN explosions that produced elements such as $\mathrm{Ca}, \mathrm{Ti}$, and $\mathrm{Zn}$ less effectively. Figure 4 shows the correlation of $[\mathrm{Zn} / \mathrm{Fe}]$ with $[\mathrm{Mg} / \mathrm{Fe}]$ in the $\mathrm{dSph}$ galaxies. Essentially, stars with lower $[\mathrm{Mg} / \mathrm{Fe}]$ exhibit lower $[\mathrm{Zn} / \mathrm{Fe}]$. One possible cause of this relation is the end result of the yields affected by differential stellar rotation; stars 


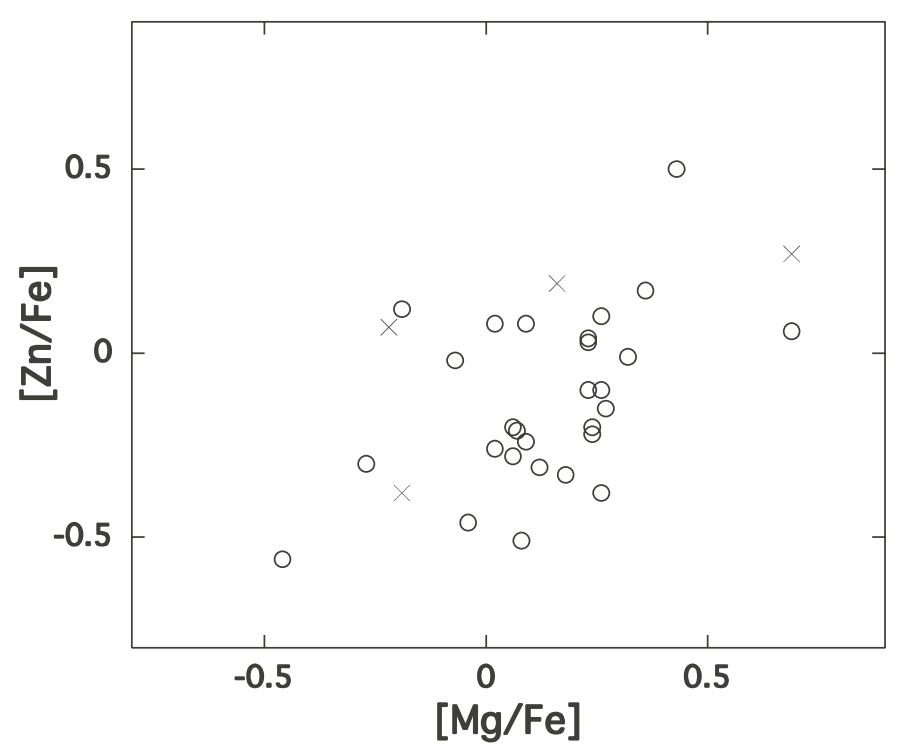

Fig. 4. $[\mathrm{Zn} / \mathrm{Fe}]$ as a function of $[\mathrm{Mg} / \mathrm{Fe}]$ for $\mathrm{dSph}$ stars (open circles, Shetrone et al. 2001, 2003; crosses, Geisler et al. 2005).

exhibiting lower $[\mathrm{Mg} / \mathrm{Fe}]$ and $[\mathrm{Zn} / \mathrm{Fe}]$ may be born from the interstellar matter enriched by massive stars with less rotation. A large scatter might be attributable to the different production processes between two elements as stated above.

Theoretical calculations have predicted that the metallicity is a key factor to control the stellar rotation in such a way that low-metallicity stars have higher rotational velocities as a result of less angular momentum loss during main-sequence evolution (Meynet \& Maeder 2000; Maeder \& Meynet 2001). However, recent direct observations of $V \sin i$ for O-type stars in the Magellanic Clouds (MCs) which have low metallicities reveal that distributions of rotational velocities for MC stars and Galactic stars are similar, and do not support these predictions (Penny et al. 2004). As discussed, elemental abundances in the LMC also have no signature of stellar rotation different from Galactic stars. The dSph galaxies of which the metallicity is much lower than those of MCs and the Milky Way are predicted to have stars with less rotation in an opposite sense to the metallicity effect. It might suggest that there is another environmental factor that regulates the stellar rotation. This factor probably would determine initial rotational velocities resulting from the angular momentum loss during star formation through the interaction between an accretion disk and star by regulating the strength of the magnetic coupling to the disk or the lifetime of the accretion disk.

\section{Conclusions}

The $[\alpha / \mathrm{H}]$ vs. $[\mathrm{Fe} / \mathrm{H}]$ diagrams where $\alpha$ represents $\mathrm{Mg}$ or an average of $\alpha$-elements for stars in the dSph galaxies (i.e., Draco, Ursa Minor, Sextan, Sculptor, Carina, Fornax, and Leo I) present no compelling evidence for the contribution of $\mathrm{SNe}$ Ia to stellar abundances in low $-\alpha / \mathrm{Fe}$ stars, in contrast to the Sagittarius galaxy and the LMC. It might suggest that massive dwarf galaxies with a mass of several $10^{8}-10^{9} M_{\odot}$ retain the ejecta of SNe Ia in their gravitational potential well and the interstellar matter in these galaxies is enriched by $\mathrm{SNe}$ Ia, while this is not the case for lower mass dSph galaxies. In contrast to this model of the SNe Ia ejecta, sufficient gas should always cool down the ejecta of SNe II and prevent them from escaping from galaxies during star formation, followed by galactic wind due to an accumulated SN explosion energy.

The abundance features for other elements in dSph stars do not support the lack of massive SNe II as well as contributions from $\mathrm{SNe}$ Ia as a source of this low- $\alpha$ signature. Rather, the deficiencies in the abundances of $\alpha$-elements and $\mathrm{Zn}$ in the dSph stars appear more likely to reflect the heavy-element yields of massive stars with less rotation compared to the solar neighborhood stars, although what makes such a difference in stellar rotation is a open question. Direct observations of $V \sin i$ of stars in dSph galaxies are needed to substantiate these implications. A young population, the so-called blue plume, in the Sagittarius galaxy could be a prime candidate for measuring stellar rotation.

This hypothesis is speculative and requires further investigation, particularly with respect to the precise effects of rotation on nucleosynthesis for both pre-SN massive stars and SN explosions. The possible environmental factors promoting such rotation should also be studied.

Acknowledgements. The author is grateful to the referee P. Bonifacio for useful comments that helped improve this paper.

\section{References}

Alves, D. R., \& Nelson, C. A. 2000, ApJ, 542, 789

Audouze, J., \& Silk, J. 1995, ApJ, 451, L49

Bonifacio, P., Sbordone, L., Marconi, G. P., et al. 2004, A\&A, 414, 503

Cayrel, R., Depagne, E., Spite, M., et al. 2004, A\&A, 416, 1117

Chen, Y. Q., Nissen, P. E., \& Zhao, G. 2004, A\&A, 425, 697

Cohen, J. 2004, AJ, 127, 1545

Dolphin, A. E., Weisz, D. R., Skillman, E. D., \& Holtzman, J. A. 2005, in Resolved Stellar populations, ed. D. Valls-Gabaud, \& M. Chavez, ASP Conf. Ser., in press

Edvardsson, B., Andersen, J., Gustafsson, B., et al. 1993, A\&A, 275, 101

Feltzing, S., Bensby, T., \& Lundström, I. 2003, A\&A, 397, L1

Fryer, C. L., \& Warren, M. S. 2004, ApJ, 601, 391

Geisler, D., Smith, V. V., Wallerstein, G., Gonzalez, G., \& Charbonnel, C. $2005, \mathrm{AJ}, 129,1428$

Gilmore, G., \& Wyse, R. F. G. 1991, ApJ, 367, L55

Grebel, E. K., \& Gallagher, J. S. 2004, ApJ, 601, L89

Hanson, R. B., Sneden, C., Kraft, R. P., \& Fulbright, J. 1998, AJ, 116, 1286

Heger, A., \& Langer, N. 2000, ApJ, 544, 1016

Heger, A., Langer, N., \& Woosley, S. E. 2000, ApJ, 528, 368

Hill, V., Andrievsky, S., \& Spite, M. 1995, A\&A, 293, 347

Hill, V., François, P., Spite, M., Primas, F., \& Spite, F. 2000, A\&A, 364, L19

Hirschi, R., Meynet, G., \& Maeder, A. 2004, A\&A, 425, 649

Hirschi, R., Meynet, G., \& Maeder, A. 2005, A\&A, 433, 1013

Ikuta, C., \& Arimoto, N. 2002, A\&A, 391, 55

Ishimaru, Y., \& Wanajo, S. 1999, ApJ, 511, L33

Korn, A. J., Keller, S. C., Kaufer, A., et al. 2002, A\&A, 385, 143

Lanfranchi, G. A., \& Matteucci, F. 2004, MNRAS, 351, 1338

Law, D. R., Johnston, K. V., \& Majewski, S. R. 2005, ApJ, 619, 823

Maeda, K., \& Nomoto, K. 2003, ApJ, 598, 1163 
Maeder, A., \& Meynet, G. 2001, A\&A, 373, 555

Maeder, A., \& Meynet, G. 2005, in Proc. of the ESO-Arcetri conference, Chemical abundances and mixing in stars in the Milky Way and its satellites, ed. L. Pasquini, \& S. Randich, in press

Mateo, M. L. 1998, ARA\&A, 36, 435

Matteucci, F., \& Greggio, L. 1986, A\&A, 154, 279

McWilliam, A. 1997, ARA\&A, 35, 503

McWilliam, A., \& Smecker-Hane, T. A. 2003, ApJ, 592, L21

McWilliam, A., Preston, G. W., Sneden, C., \& Searle, L. 1995, AJ, 109,2757

Meynet, G., \& Maeder, A. 2000, A\&A, 361, 101

Monaco, L., Bellazzini, M., Bonifacio, P., et al. 2005, A\&A, 441, 141

Nagataki, S., Hashimoto, M., Sato, K., \& Yamada, S. 1997, ApJ, 486, 1026

Nissen, P. E., Chen, Y. Q., Aplund, M., \& Pettini, M. 2004, A\&A, 415, 993

Pagel, B. E. J. 1997, Nucleosynthesis and Chemical Evolution of Galaxies (Cambridge: Cambridge Univ. Press)

Penny, L. R., Sprague, A. J., Seago, G., \& Gies, D. R. 2004, ApJ, 617, 1316

Robertson, B., Bullock, J. S., Font, A. S., Johnston, K. V., \& Hernquist, L. 2005, ApJ, 632, 872

Sbordone, L. 2005, in Proc. of the ESO-Arcetri conference, Chemical abundances and mixing in stars in the Milky Way and its satellites, ed. L. Pasquini, \& S. Randich, in press
Shetrone, M. D., Côté, P., \& Sargent, W. L. 2001, ApJ, 548, 592 Shetrone, M. D., Venn, K. A., Tolstoy, E., et al. 2003, AJ, 125, 684 Shigeyama, T., \& Tsujimoto, T. 1998, ApJ, 507, L135 Shigeyama, T., \& Tsujimoto, T. 2003, ApJ, 598, L47

Smith, V. V., Hinkle, K. H., Cunho, K., et al. 2002, AJ, 124, 3241

Thielemann, F.-K., Hashimoto, M., \& Nomoto, K. 1990, ApJ, 349, 222

Tolstoy, E. 2005, in Near-field Cosmology with Dwarf Elliptical Galaxies, ed. H. Jerjen, \& B. Binggeli, IAU Colloq., 198, 118

Tolstoy, E., Venn, K. A., Shetrone, M., et al. 2003, AJ, 125, 707

Tolstoy, E., Irwin, M. J., Helmi, A., et al. 2004, ApJ, 617, L119

Travaglio, C., Galli, D., Gallino, R., et al. 1999, ApJ, 521, 691

Tsujimoto, T., \& Shigeyama, T. 2002, ApJ, 571, L93

Tsujimoto, T., Nomoto, K., Yoshii, Y., et al. 1995, MNRAS, 277, 945

Tsujimoto, T., Shigeyama, T., \& Yoshii, Y. 2000, ApJ, 531, L33

Umeda, H., \& Nomoto, K. 2002, ApJ, 565, 385

Van Dyk, S. D., Garnavich, P. M., Filippenko, A. V., et al. 2002, PASP, 114,1322

Venn, K. A., \& Hill, V. 2005, in From Lithium to Uranium: Elemental Tracers of Early Cosmic Evolution, ed. V. Hill, P. Francois, \& F. Primas, IAU Symp., 228, in press

Venn, K. A., Irwin, M., Shetrone, M. D., et al. 2004, AJ, 128, 1177

Wheeler, J. C., Sneden, C., \& Truran, J. W. 1989, ARA\&A, 27, 279

Yamada, S., \& Sato, K. 1994, ApJ, 434, 268 